\title{
Las redes sociales web como herramienta para gestionar información en procesos de co-creación
}

\author{
Liliana González-Palacio ${ }^{1}$, Mauricio González-Palacio ${ }^{2}$, Jairo Ortiz-Pabón ${ }^{1}$
}

ligonzalez@udem.edu.co,magonzalez@udem.edu.co, hortiz@udem.edu.co

${ }^{1}$ Ingeniería de sistemas- Facultad de ingeniería- Universidad de Medellín, Carrera 87 Nro. 30-65, 50026, Medellín, Colombia

${ }^{2}$ Ingeniería en telecomunicaciones- Facultad de ingeniería- Universidad de Medellín, Carrera 87 Nro. 30-65, 50026, Medellín, Colombia

DOI: 10.17013/risti.24.57-68

Resumen: Los procesos de innovación abierta emplean la co-creación de valor para viabilizar la interacción y colaboración entre agentes internos y externos a la organización. El uso de las tecnologías de información y comunicación hace posible que participantes distribuidos geográficamente y con limitantes de tiempo puedan aportar sus ideas en cualquier momento y lugar. Particularmente las redes sociales surgen como una alternativa interesante en la co-creación, ya que facilitan el involucramiento de colectivos identificados con las mismas necesidades y problemáticas. También estimulan el intercambio dinámico de experiencias en contextos de variada complejidad. En esta investigación se propone un protocolo para seleccionar una red social acorde a escenarios específicos de aplicación de la co-creación de valor. La iniciativa es validada mediante un caso real donde se evidencia que el uso de una red social para una construcción colectiva favorece la sinergia entre participantes heterogéneos.

Palabras-clave: Redes sociales, innovación abierta, co-creación, colaboración, interacción.

\section{Social networks as a tool to manage information in co-creation processes}

\begin{abstract}
Open innovation processes employ the co-creation of value for the interaction and collaboration between internal and external agents to the organization. The use of information and communication technologies enables stakeholders distributed geographically and with time constraints to express their ideas anywhere and anytime. Particularly, social networks emerge as an interesting alternative in co-creation, due to they facilitate the involvement of groups identified with the same needs and problems. They also encourage the dynamic exchange of experience in contexts of different kinds of complexity. This research proposes a protocol to select a social network according to specific application scenarios of cocreation of value. The initiative is validated by a real case where is evident that the use of a social network for collective construction promotes synergy between heterogeneous stakeholders.
\end{abstract}


Keywords: social networks, open innovation, co-creation, collaboration, interaction.

\section{Introducción}

En el paradigma tradicional de innovación las compañías siguen su cadena de valor ubicando al cliente en un rol pasivo. Hoy, la innovación abierta estimula la capacidad innovadora, acelera la transferencia de conocimiento e intensifica la capacidad de colaboración endógena y exógena de las empresas (Chesbrough, 2006; Enkel, Gassmann, \& Chesbrough, 2009). La innovación ahora es pensada bajo un esquema abierto (Chesbrough, 2006) donde actores y agentes externos co-crean valor en todo el proceso y son escuchados por las compañías que fabrican nuevas ofertas, las cuales a su vez reciben aportes continuos y retroalimentación.

La innovación abierta (IA) es un modelo mediante el cual las empresas hacen uso intensivo de conocimiento interno y externo, con el objetivo de potenciar y acelerar su innovación interna y expandir los mercados (Chesbrough, 2006). Esta dinámica para innovar requiere la incorporación de medios tecnológicos adecuados para la interacción (Enkel, et al., 2009). El concepto de red social ha evolucionado mostrándose como una herramienta poderosa para facilitar procesos de co-creación de valor. Se evidencian vacíos importantes al seleccionar la red social adecuada, y finalmente la elección se hace sin tener criterios claros.

En esta investigación se propone un protocolo para seleccionar una red social acorde a las necesidades específicas de un proceso de co-creación de valor. Para el efecto se inicia con el levantamiento de un referente conceptual y la revisión de literatura, que permiten analizar la problemática. Posteriormente se presenta una propuesta de solución a las carencias identificadas. La iniciativa es validada mediante un caso real.

El artículo se organiza como sigue: la sección 2 muestra el fundamento teórico que sustenta la propuesta. La sección 3 contiene el trabajo relacionado. La problemática es descrita en el apartado 4. La sección 5 detalla el protocolo para la selección de una red social apropiada acorde a un ejercicio de co-creación específico. La sección 6 describe la validación. Finalmente se presentan las conclusiones, trabajo futuro, agradecimientos y referencias.

\section{Marco teórico}

Esta investigación se enmarca en los conceptos de innovación, co-creación, y redes sociales. A continuación se explican brevemente (Gonzalez, González, Echeverri, \& Urrego, 2013; González \& González, 2015):

- Innovación: según el manual de Oslo se define como la implementación de un producto (bien, servicio, proceso) nuevo o significativamente mejorado que se incorpora en el mercado (OECD, 2005).

- Co-creación: hace alusión a la generación conjunta de valor a partir de la interacción entre agentes internos y externos a la organización. Esto se potencia mediante los principios de diálogo continuo, acceso a la información, riesgo/ beneficio y transparencia (Prahalad \& Ramaswamy, 2004). Este concepto 
está íntimamente ligado con la materialización de la innovación abierta en las organizaciones. Las características enunciadas exigen un manejo adecuado de aspectos tecnológicos y de seguridad, al tiempo que requieren un alto nivel de colaboración y comunicación entre los participantes (Ormaetxe, 2008).

- Redes sociales: originalmente hacen referencia a una reunión de personas, conocidas o desconocidas, que interactuarán entre sí, redefiniendo al grupo y retroalimentándolo en lo que podría asimilarse a una dinámica de trabajo, o bien a una estructura auto-organizada de personas compartiendo, manipulando y transformando información colaborativamente (Raghavan, 2002). Las redes sociales pueden definirse, como asociaciones de personas ligadas por motivos heterogéneos y que conforman una estructura compuesta por nodos unidos entre ellos por más de un tipo de relación (Requena, 2008). Los usuarios frecuentes de Facebook, o de otra red de características similares, suelen argumentar que se trata de un medio eficiente para mantener o renovar vínculos con amigos, familiares, instituciones u otros grupos de interés; pero más allá de esa percepción, algunos investigadores han equiparado el alcance de esa red social con el de un complejo sistema abierto y en construcción permanente que involucra a colectivos identificados con las mismas necesidades y problemáticas, donde además se estimula el intercambio dinámico de experiencias, conocimientos y muchos otros aspectos de la vida en contextos de variada complejidad (Anria, 2009). En esa misma dirección, esta dinámica de interacción permite a grupos e individuos, a través del mismo modelo de organización y disposición de infinidad de informaciones allí contenidas, potenciar sus recursos cognitivos (Caldevilla, 2010; Maamar \& Badr, 2009; Tavante \& Coutinho, 2011). Las redes sociales se vislumbran como una poderosa herramienta para facilitar la co-creación.

\section{Trabajo relacionado}

Esta sección da cuenta de una exploración sobre el uso de las redes sociales web en procesos de innovación abierta y co-creación de valor, o sectores afines.

Los países cuyas empresas están más avanzadas en el uso de las herramientas Web con fines de innovación colectiva y como parte de su estrategia empresarial son Estados Unidos, Reino Unido, Alemania, China e India (Nafría, 2008). Mientras que el entretenimiento y el turismo son algunos de los sectores que más aprovechan las ventajas que ofrece este tipo de tecnología comunicacional.

Profundizando en algunas iniciativas, (Cohen \& Clemens, 2005) proponen una red social web para facilitar la colaboración creativa. La idea fue validada en la empresa consultora Razorfish, dedicada servicios de fidelización de marca. Dentro de su estudio se involucraron cincuenta diseñadores de interfaces, arquitectos de información y en general, ingenieros de sistemas. Como propuesta se creó una red social web denominada "Peers" para promover internamente la solución al problema organizacional en el cual la información no está visible a todos los integrantes del equipo, debido a la carencia de interacciones entre los mismos. Dicha red tiene un formato de blog compartiendo espacios de trabajo, además incluye una forma para votar por los aportes de cada usuario (clasificándolos de acuerdo a su relevancia). 
(Lim, Damian, \& Finkelstein, 2011) diseñaron una red social para apoyar los procesos de captura de requisitos (centrados en los usuarios) en diseño de software. Lograron evidenciar una mejora en la comunicación entre todos los participantes del diseño de un nuevo proyecto, en términos de fluidez, efectividad y trazabilidad.

(Tomsic \& Suthers, 2005) llevaron a cabo un estudio observando las herramientas de interacción comunes dentro del departamento de policía de Honolulú (teléfono, correo electrónico y cara a cara). Luego analizaron cómo la inclusión de una red social mejora la comunicación y encontraron un aumento significativo.

(Murphy, Sheth, Kaiser, \& Wilcox, 2008) sostienen que las herramientas para trabajo colaborativo en labores científicas se basan en la capacidad de compartir información, mas no conocimiento explícito. Argumentan además que las redes sociales son un medio ideal para alcanzar una comunicación más efectiva entre los participantes. Diseñaron la herramienta GenSpace cuya estructura tipo red social incluye mensajería instantánea, sugerencias de posibles contactos y clasificación de perfiles (básicos y expertos).

(Althoff, Jindal, \& Leskovec, 2017) identifican en el uso de las redes sociales WEB seis características fundamentales: (1) son dinámicas, puesto que los contenidos se actualizan de forma constante; (2) son colaborativas, pues se elaboran por un grupo de personas; son simples e intuitivas; pueden utilizarse sin necesidad de instalar nada en el ordenador, ya que la web es la plataforma; (5) poseen un entorno amigable e interactivo y los usuarios tienen capacidad de gestionar qué, cuándo y cómo publicar.

(Aiello \& Ruffo, 2012) se concentran en la seguridad en redes sociales y destacan la necesidad de potenciarla este aspecto facilitando interacciones confiables entre participantes. Así diseñan un framework para ser incluido en una red social, destacando atributos tales como la confidencialidad, la privacidad de las contribuciones, entre otros.

El estudio de Yang y Chen se enfoca en demostrar que las redes sociales facilitan el intercambio de conocimientos posibilitando que los participantes encuentren contenido relevante y compartan alrededor de temas específicos (Yang \& Chen, 2008). Los autores también descubren un incremento en la creatividad por tratarse de una actividad social en la cual se genera conocimiento colectivo mediante la interacción. Además, proponen una arquitectura P2P para configurar redes sociales web.

(Anria, 2009) relaciona las tecnologías más usadas en la interacción a través de redes sociales, además de categorizar la comunicación mediada por el computador en: Uno a uno (Ejemplo: Mensajería instantánea para interacción privada), uno a muchos (Ejemplo: páginas web o blogs) y muchos a muchos (Ejemplo: wikis o Whiteboards). Este autor también enuncia las características fundamentales de una red social web y por último enuncia los beneficios y riesgos asociados al uso de estas herramientas en un entorno empresarial.

Dall'Asta y otros estudian la interacción en redes sociales para hacer analogías con la teoría de juegos (Dall'Asta, Marsili, \& Pin, 2012). Se concentran en las diferentes topologías de red y su conformación en términos de grafos, para asociar estas estructuras a características que facilitan la interacción.

La innovación es esencial para que las empresas sobrevivan en la economía global de hoy. La co-creación es una estrategia donde clientes y proveedores participan en el proceso de innovación. Los medios sociales son una herramienta perfecta para co-crear 
valor ya que promueven la comunicación entre la organización y el cliente de una forma fluida e intuitiva (Halale, Gangadharan, \& Uden, 2015).

\section{Problemática}

Las organizaciones están empleando cada vez más las redes sociales en los negocios. Este fenómeno también atrae la atención de los investigadores. Los medios de comunicación social se utilizan en la co-creación de valor para permitir a los consumidores tomar un papel activo y co-crear valor para las empresas. Sin embargo, la forma en que los medios sociales pueden mejorar la capacidad de co-creación de valor de una empresa no ha sido estudiada en la misma medida (Wang, Li, \& Suomi, 2016).

Es evidente que las redes sociales web son herramientas usadas en diversos sectores productivos para facilitar la interacción de participantes que comparten intereses, gustos y se encuentran distribuidos geográficamente. Al explorar el dominio de la co-creación mediante redes sociales web no se evidenciaron propuestas para elegir de una manera sistemática la red social que mejor se ajusta a un escenario particular.

De allí surge la idea de proveer un protocolo de selección de una red social con fines de co-creación, empleando para el efecto un conjunto de criterios que hagan el ejercicio replicable y argumentable. Esto debería mejorar indicadores como la cantidad de ideas recopiladas durante la co-creación de valor.

\section{Solución propuesta}

Se propone el siguiente método (figura 1) para la selección de una red social durante un ejercicio de co-creación.

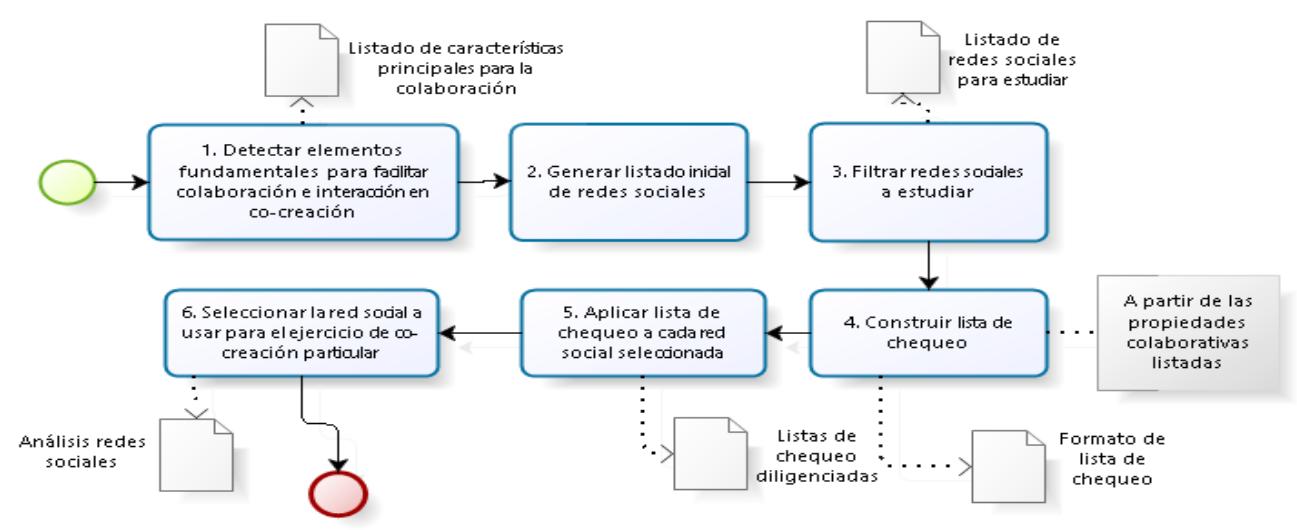

Figura 1 - Proceso para la selección de redes sociales en ejercicios de co-creación 
Para la detección de las características de trabajo colaborativo, y sin perder de vista las particularidades de un ejercicio de co-creación, se sugiere incluir las siguientes características (González, González, \& Urrego, 2013): organización y categorización de contactos (compartir, importar/exportar, conformación de grupos); sugerencias y recomendaciones de contactos (Cohen \& Clemens, 2005; Lim, et al., 2011); generación de comentarios, y trazabilidad sobre éstos; intercambio de archivos de diferentes tipos (Murphy, et al., 2008); modificación en línea de los archivos compartidos (Aiello \& Ruffo, 2012); gestión de versiones de los archivos modificados (Reinhardt, Moi, \& Varlemann, 2009; Yang \& Chen, 2008); notificaciones a los usuarios sobre cambios en los archivos; generación de blogs; programación de eventos (una vez, periódicos, rango de fechas); notificaciones y recordatorios de los eventos usando varios medios (correo, mensajes al móvil, ventanas emergentes, etc); generación de listas de distribución (Cohen \& Clemens, 2005; Lim, et al., 2011); despliegue de publicidad; despliegue de calendario (con posibilidad de compartir con otros usuarios); realización de video-llamadas y webinar; intercambio de mensajes de forma síncrona y asíncrona; búsqueda de elementos (archivos, eventos, contactos); selección de estados (disponible, ocupado, ausente, desconectado, etc); esquema de calificación de usuarios de acuerdo a participación; mecanismo de premiación o bonificación para usuarios (Cohen \& Clemens, 2005; Lim, et al., 2011); exportación e integración con otras redes sociales; publicación de contenidos (Reinhardt, et al., 2009; Yang \& Chen, 2008); soporte para ambientes móviles; esquema de privacidad adecuado.

El siguiente paso es seleccionar el conjunto de redes sociales desde donde se elegirá una para hacer co-creación. Esta no es una labor trivial dada la cantidad exorbitante de redes sociales existentes. Para el efecto se sugiere revisar los listados y reportes ofrecidos por instituciones (en diversos países) como:

\begin{tabular}{ll}
\hline Institución & Descripción \\
\hline Elogia IPSOFACTO (Elogia- & $\begin{array}{l}\text { Son expertos en marketing para comercio electrónico. En conjunto con } \\
\text { Ipsofacto, 2015) }\end{array}$ \\
& $\begin{array}{l}\text { IAB Research Institute hacen estudios periódicos para evaluar cómo } \\
\text { han evolucionado los principales indicadores de las redes sociales más } \\
\text { relevantes. }\end{array}$ \\
\hline & $\begin{array}{l}\text { Se encargan de hacer investigación de marketing en Internet, ofreciendo } \\
\text { servicios de información para muchas de las mayores empresas de Internet. } \\
\text { Los análisis son hechos en diversos países, atendiendo el comportamiento } \\
\text { variado de los usuarios. }\end{array}$ \\
\hline EBizMBA (eBizMBA, 2015) & Rango generado a partir del Alexa Ranking Global Traffic y EE.UU. ranking \\
\hline
\end{tabular}

Tabla 1 - Reportes sobre popularidad en redes sociales web

Se pueden usar criterios como la frecuencia de uso de la red social, el tipo de interacción que facilita, los públicos que usan la herramienta a menudo, entre otros, para hacer la preselección de redes sociales a explorar con mayor detalle (Roch \& Mosconi, 2016).

A este subconjunto de herramientas para la interacción se le debe aplicar el checklist que se indica en la tabla 2: 


\begin{tabular}{ll}
\hline Categoría & Descripción \\
\hline 1 & $\begin{array}{l}\text { Espacio compartido de trabajo. Preguntas asociadas: ¿Es posible compartir documentos, } \\
\text { compartir notas, compartir medios (videos, imágenes, presentaciones), generar blogs y } \\
\text { wikis? }\end{array}$ \\
\hline 2 & $\begin{array}{l}\text { Calendario compartido. Preguntas asociadas: ¿Se puede tener una agenda común, generar } \\
\text { eventos compartidos en grupos, realizar seguimiento a una tarea particular a través de } \\
\text { notificaciones automáticas? }\end{array}$ \\
\hline 3 & $\begin{array}{l}\text { Mensajería y videoconferencia Preguntas asociadas: ¿Se cuenta con un sistema similar al } \\
\text { correo electrónico?; cexiste un concepto similar al de un foro?; ¿existe un chat online? ¿El } \\
\text { chat permite videoconferencia? }\end{array}$ \\
\hline 4 & $\begin{array}{l}\text { Configuración de perfiles / privacidad. Preguntas asociadas: ¿El usuario puede configurar } \\
\text { su perfil propio, unirse a un grupo de interés particular y/o restringir a otros el acceso a su } \\
\text { perfil? }\end{array}$ \\
\hline 5 & $\begin{array}{l}\text { Recuperación de información. Preguntas asociadas: ¿Es posible generar etiquetas (o tags) } \\
\text { de la información publicada por el usuario sobre el contenido compartido?; ¿Es posible } \\
\text { organizar la información de acuerdo a una marca de hora y fecha? }\end{array}$ \\
\hline 6 & $\begin{array}{l}\text { Herramientas para medir la colaboración. Preguntas asociadas: ¿Existe un mecanismo } \\
\text { explícito para aprobar o desaprobar un pensamiento, conducta, aporte o idea de otro } \\
\text { usuario?; ¿El aporte puede ser re-enviado? }\end{array}$ \\
\hline $\begin{array}{l}\text { Interacción entre usuarios. Preguntas asociadas: ¿Se pueden compartir contactos? ¿se } \\
\text { generan sugerencias de posibles contactos? }\end{array}$
\end{tabular}

Tabla 2 - Lista de chequeo para la selección de redes sociales web

Se debe explorar la presencia o ausencia de cada categoría de la lista de chequeo anterior y de esta manera encontrar cuál de las redes sociales exploradas logra agrupar mayor cantidad de características favorables para un ejercicio de co-creación. De esta forma se determinará la herramienta a usar.

Siguiendo este método se tendrá un subconjunto de redes sociales con un ranking que indica cuál de ellas favorece más el trabajo colaborativo.

\section{Validación}

El escenario de validación fue un ejercicio de co-creación para aportar ideas sobre los elementos esenciales de un modelo de comercialización tecnológica en la región antioqueña (en Colombia). Los participantes fueron expertos en comercialización de tecnología de diversos países. Se requería elegir una herramienta adecuada para interacción virtual. Junto con los interesados en el ejercicio se definió que una red social web cubría los requerimientos para facilitar el intercambio de ideas. Para elegir cuál red social se usó el protocolo presentado en este artículo. Algunas características adicionales del escenario se indican a continuación:

- Se buscó proporcionar un medio de interacción entre expertos a escala global facilitando el espacio virtual para compartir experiencias y conocimiento informal entre los participantes. 
- Los participantes estaban distribuidos geográficamente en países distantes.

- Se usó el inglés como idioma para la interacción.

Guiados por el método presentado anteriormente, en el primer paso se retomó el listado de características colaborativas sugerido sin encontrar otro aspecto adicional.

En el segundo paso se buscó un subconjunto de redes sociales representativas. Para ello se revisaron 12 reportes de las instituciones enunciadas en el método objeto de este artículo (Elogia IPSOFACTO en conjunto con IAB Research Institute, ComScore en países como Brasil, México, Argentina, Chile, Venezuela, Perú (Yanqui \& Quispe, 2015), Colombia, EBizMBA). Se usó como criterio de selección las redes que tuvieran un porcentaje mayor de aparición en los reportes analizados, tal como se indica en la tabla 3:

\begin{tabular}{llllll}
\hline$\#$ & Red social & \% aparición & $\#$ & Red social & \% aparición \\
\hline 1 & Facebook & $52.3 \%$ & 6 & MySpace & $3.8 \%$ \\
\hline 2 & Twitter & $13.8 \%$ & 7 & Badoo & $3.9 \%$ \\
\hline 3 & YouTube & $8.4 \%$ & 8 & Sonico & $3.5 \%$ \\
\hline 5 & LinkedIn & $4.6 \%$ & 9 & Fotolog & $3.4 \%$ \\
\hline & SlideShare & $4 \%$ & 10 & Google+ & $2.3 \%$ \\
\hline
\end{tabular}

Tabla 3 - Redes sociales web con mayor porcentaje de aparición

Los porcentajes se obtienen al revisar en cuantos reportes aparece cada red social elegida y normalizando los valores.

A partir de la interacción con las redes sociales del ranking anterior y usando como guía el checklist se obtuvo un listado priorizado de aquellas con mayor grado de colaboración, tal como se muestra en la tabla 4.

\begin{tabular}{llllll}
\hline$\#$ & Red social & \% colaboración & $\#$ & Red social & \% colaboración \\
\hline 1 & Google + & $100.00 \%$ & 6 & Sonico & $63.60 \%$ \\
\hline 2 & Facebook & $90.00 \%$ & 7 & Youtube & $63.60 \%$ \\
\hline 3 & SlideShare & $81.80 \%$ & 8 & Twitter & $59.10 \%$ \\
\hline 5 & LinkedIn & $77.30 \%$ & 9 & Badoo & $45.50 \%$ \\
\hline
\end{tabular}

Tabla 4 - Valoración de redes sociales web acorde a su nivel de colaboración

Los porcentajes generados dan cuenta de una razón entre el número de respuestas positivas encontradas con respecto al número total de preguntas. Por ejemplo, en Google+ todas las herramientas colaborativas están presentes, mientras que en Fotolog aproximadamente tres de cada diez herramientas son incorporadas. 
Los encargados del ejercicio de co-creación para el modelo de comercialización atendieron la sugerencia de usar la red social google+ obteniendo resultados muy positivos al igual que comentarios valiosos para la construcción colectiva. Algunos de ellos se enuncian seguidamente:

- Cliff Zintgraff, CEO at DaVinci Minds: "I believe the most important issue is building a structure that allows the individual IP holders (universities, etc.) to maintain control of university policy issues, while agreeing to share resources for commercialization analysis. I think there are selected policy issues, like inventor royalty agreements, that universities will insist on keeping. Universities' comfort level that their interests are protected will be key to gaining their enthusiastic support".

- Alex Smeets, CEO at Cambridge Innovation Solutions: "Congratulations on starting this new initiative - by working together I think you'll achieve a lot more than if every organization in the region tried to do its own technology commercialization in isolation. I believe that the key to successful technology commercialization is to develop the best possible understanding of all the potential applications and markets for the technology. This requires creative, multidisciplinary thinking (to explore the possible applications) as well as a very practical, hands-on approach to obtaining information from the market (typically by talking to parties in the market, not by relying on existing market research reports or databases). If you do this well, then the way forward becomes much clearer and you'll find it much easier to obtain support and investment. I would therefore suggest that you consider developing a joint market exploration capability".

Es de anotar que la red social con mayores características que facilitan la co-creación no es necesariamente la más popular (casi de google+ y facebook).

\section{Conclusiones}

En este artículo se propuso un protocolo para seleccionar una red social acorde a escenarios específicos de aplicación de la co-creación de valor. La idea surge al detectar un vacío en la revisión de literatura. Actualmente las organizaciones que desean hacer co-creación con sus clientes eligen la herramienta tecnológica a usar de forma arbitraria, con las consecuentes dificultades para replicar ejercicios exitosos y evitar aquellos que no arrojaron resultados positivos.

Esta iniciativa se constituye en una herramienta útil para las organizaciones que requieran directrices sobre la forma de facilitar la interacción de participantes heterogéneos como clientes, competidores, equipos de $\mathrm{I}+\mathrm{D}$, personal de mercadeo, entre otros. Existe un sinnúmero de beneficios resultantes al integrar las redes sociales, entre ellos la incorporación de clientes y personal externo e interno de una manera efectiva. Lo anterior a partir de la riqueza de las interacciones que ofrecen.

El uso de redes sociales web a nivel empresarial tiene beneficios tales como la ampliación de contactos, el aceleramiento de procesos de negocio, la mejora en las relaciones con los clientes y la facilidad para reclutar personal de alto nivel (Anria, 2009; Molina, 2001), 
además de facilitar la innovación a través de funcionalidades usables y atractivas para cualquier usuario (Ormaetxe, 2008).

En un mundo empresarial donde la presión competitiva está en constante aumento, las empresas están tratando de diferenciarse continuamente. La llegada de las tecnologías Web 2.0, como las redes sociales, permitió a las empresas comunicarse e interactuar con los consumidores y usuarios en línea con el fin de recopilar información y realizar tareas de I + D, marketing y ventas (Roch \& Mosconi, 2016).

La iniciativa fue validada con un caso real donde se evidenció que el uso de una red social para una construcción colectiva favorece la sinergia entre participantes heterogéneos. Sin duda alguna, en un mundo cambiante y dependiente de la tecnología, las redes sociales propician procesos colectivos, colaborativos e interactivos. Se trata de herramientas poderosas en otros dominios diferentes al ocio y el entretenimiento.

\section{Trabajo futuro}

Como trabajo futuro se plantea la identificación y recomendación de las redes sociales más apropiadas para usar de acuerdo a escenarios pre-configurados de co-creación. Por ejemplo, las redes masivas como Facebook y Google+ son útiles para hacer co-creación durante la identificación de oportunidades y generación de ideas en un ciclo de innovación, mientras que LinkedIn (la cual posee herramientas de gestión de proyectos) podría ser usada en ejercicios de co-creación asociados a etapas más avanzadas tales como el diseño o las pruebas.

También vale la pena explorar la posibilidad de proponer una nueva red social especializada para la co-creación de valor. Esta nueva red integraría lo mejor de las redes sociales existentes. También podría pensarse en usar el mismo framework que poseen otras redes sociales (por ejemplo, Facebook), siendo éste mejorado a través de complementos orientados a facilitar las interacciones entre interesados.

Otro trabajo en curso es la exploración de otras redes sociales, y la puesta en marcha del protocolo propuesto en diversos ejercicios reales para verificar su efectividad.

\section{Referencias}

Aiello, L. M., \& Ruffo, G. (2012). LotusNet: Tunable privacy for distributed online social network services. Computer Communications, 35(1), 75-88. doi: 10.1016/j. comcom.2010.12.006

Althoff, T., Jindal, P., \& Leskovec, J. (2017). Online actions with offline impact: How online social networks influence online and offline user behavior. In Proceedings of the 1oth ACM International Conference on Web Search and Data Mining (WSDM 2017). Cambridge, UK.

Anria, S. (2009). The impact of Social Networking 2.0 on organisations. Electronic Library, 27(6), 906-918. doi: 10.1108/02640470911004020

Caldevilla, D. (2010). Las Redes Sociales: Tipología, uso y consumo de las redes 2.0 en la sociedad digital actual. Documentación de las Ciencias de la Información, 33, 45-68. 
Cohen, T., \& Clemens, B. (2005). Social networks for creative collaboration. In Proceedings of the 5 th conference on Creativity \& cognition (C\&C'O5). London, UK. ACM

ComScore. (2015). Audience Analytics. Retrieved from http://www.comscore.com/ Products/Audience-Analytics

Chesbrough, H. (2006). Open innovation: a paradigm for understanding industrial innovation. Oxford, UK: Oxford University Press.

Dall'Asta, L., Marsili, M., \& Pin, P. (2012). Collaboration in social networks. In Proceedings of the National Academy of Sciences of the United States of America, New York, United States, 109(12), 4395-4400.

eBizMBA. (2015). Top 15 Most Popular Social Networking Sites 2016. Retrieved from http://www.ebizmba.com/articles/social-networking-websites

Elogia-Ipsofacto. (2015). VI Estudio de Redes Sociales de IAB Spain Retrieved from http:// www.informeticplus.com/estudio-anual-redes-sociales-2017-iab-spain-elogia.

Enkel, E., Gassmann, O., \& Chesbrough, H. (2009). Open R\&D and open innovation: exploring the phenomenon. R\&D Management, 39(4), 311-316.

Gonzalez, L., González, M., Echeverri, J., \& Urrego, G. (2013). Method of interaction in open innovation processes incorporating ubiquitous environments and web 2.0 social networks: A baseline architecture. In Proceedings the 8th Iberian Conference on Information Systems and Technologies (CISTI 2013), IEEE.

González, M., \& González, L. (2015). La co-creación como estrategia para abordar la gobernanza de TI en una organización. RISTI-Revista Ibérica de Sistemas e Tecnologias de Informação (15), 01-16.

González, M., González, L., \& Urrego, G. (2013). Survey of interaction in web 2.0 social networks and its application to support open innovation processes. In Proceedings the 8th Iberian Conference on Information Systems and Technologies (CISTI 2013), IEEE.

Halale, S., Gangadharan, G., \& Uden, L. (2015). Enhancing Cocreation Using Social Media. IT Professional, 17(2), 40-45.

Lim, S. L., Damian, D., \& Finkelstein, A. (2011). StakeSource2. o: using social networks of stakeholders to identify and prioritise requirements. In Proceedings of the 33rd international conference on Software engineering (ICSE'2011). Honolulu, Hawaii.

Maamar, Z., \& Badr, Y. (2009). Social networks as a service in modern enterprises. In Proceedings of the International Conference on the Current Trends in Information Technology (CTIT 2009). Dubai, United Arab Emirates. IEEE

Molina, J. L. (2001). El análisis de redes sociales. Barcelona: Editorial Bellaterra.

Murphy, C., Sheth, S., Kaiser, G., \& Wilcox, L. (2008). GenSpace: Exploring social networking metaphors for knowledge sharing and scientific collaborative work. In Proceedings of the 23rd IEEE/ACM International Conference on Automated Software Engineering (ASE 2008). L'Aquila, Italy. IEEE. 
Nafría, I. (2008). Web 2.o. El usuario, el nuevo rey de Internet. Barcelona: Ediciones Gestión 2000.

OECD. (2005). Oslo Manual: Guidelines for collecting and interpreting innovation data ( $3^{\mathrm{a}}$ ed.). Paris: OECD Publishing.

Ormaetxe, J. I. (2008). Redes sociales e innovación abierta: apuntes críticos. Telos: Cuadernos de comunicación e innovación (76), 114-117.

Prahalad, C., \& Ramaswamy, V. (2004). Co-creation experiences: the next practice in value creation. Journal of interactive marketing, 18(3), 5-14.

Raghavan, P. (2002). Social networks: from the Web to the enterprise. IEEE Internet Computing, 6(1), 91-94.

Reinhardt, W., Moi, M., \& Varlemann, T. (2009). Artefact-Actor-Networks as tie between social networks and artefact networks. In Proceedings of the 5th International Conference on Collaborative Computing: Networking, Applications and Worksharing (CollaborateCom 2009). Edinburgh, Great Britain. IEEE.

Requena, S. (2008). El modelo constructivista con las nuevas tecnologías: aplicado en el proceso de aprendizaje. RUSC. Universities and Knowledge Society Journal, 5(2), 26-35.

Roch, J., \& Mosconi, E. (2016). The use of social media tools in the product life cycle phases: A systematic literature review. In Proceedings of the 49th Hawaii International Conference on System Sciences (HICSS 2016). Koloa, USA.

Tavante, H. C. A., \& Coutinho, M. P. (2011). Web social networks meters and business usage analisys. In Proceedings of the International Conference on Computational Aspects of Social Networks (CASoN 2011). Salamanca, Spain.

Thrift, N. (2006). Re-inventing invention: new tendencies in capitalist commodification. Economy and Society, 35(2), 279-306.

Tomsic, A., \& Suthers, D. (2005). Effects of a Discussion Tool on Collaborative Learning and Social Network Structure within an Organization. In Proceedings of the 38th Annual Hawaii International Conference on the System Sciences (HICSS '2005). Big Island, HI, USA.

Wang, P., Li, H., \& Suomi, R. (2016). Value Co-Creation in Business via Social Media: a Technology Affordance Approach. In Proceedings of the Pacific Asia Conference on Information Systems (PACIS 2016). Chiayi, Taiwan.

Yang, S. J. H., \& Chen, I. Y. L. (2008). A social network-based system for supporting interactive collaboration in knowledge sharing over peer-to-peer network. International Journal of Human-Computer Studies, 66(1), 36-50. doi: 10.1016/j. ijhcs.2007.08.005

Yanqui, F., \& Quispe, M. (2015). Usode las redes sociales en el Perú. Investigación Andina, 15(2). 